\title{
Outburst of the unique X-ray transient CI Cam and its impact on the system
}

\author{
V. Šimon, ${ }^{1}$ C. Bartolini, ${ }^{2}$ A. Guarnieri, ${ }^{2}$ A. Piccioni ${ }^{2}$ and D. Hanžl ${ }^{3}$ \\ ${ }^{1}$ Astronomical Institute, Academy of Sciences, 25165 Ondřejov, Czech Republic \\ ${ }^{2}$ Dipartimento di Astronomia, Università di Bologna, via Ranzani 1, 40127 Bologna, Italy \\ ${ }^{3}$ N. Copernicus Observatory and Planetarium, Kraví hora 2, 61600 Brno, Czech Republic
}

\begin{abstract}
We argue that the outburst of CI Cam (XTE J0421+560), probably containing a black hole, was caused by the thermal instability of the accretion disk. Applying the model of King \& Ritter (1998), we obtain a realistic disk mass and radius. The differences from soft X-ray transients belonging to the low-mass X-ray binaries can be explained if the disk in CI Cam heats up an extended envelope and/or a strong jet is formed. We resolve several spectral components in the optical colors in quiescence after the outburst; they vary in a complicated way during a possible 1350 d cycle.

We find indications that the variations of the source of the optical light can be related to those of the X-ray source in quiescence. The accretion disk seems to refill at present. Nowadays, only non-periodic intra-night optical $(\sim 0.02 \mathrm{mag})$ fluctuations are present. As regards the absence of coherent changes in the optical band, we point out the similarities in the situation of CI Cam and the microquasar LS5039/RX J1826-1450.
\end{abstract}

Keywords. Accretion, accretion disks - instabilities - stars: variables: other - X-rays: binaries

\section{Introduction and results}

CI Cam, an optical counterpart of the unique X-ray transient XTE J0421+560 (e.g. Frontera et al. 1998), is a remarkable system consisting of a $\mathrm{B}[\mathrm{e}]$ supergiant and probably a black hole (BH, e.g. Belloni et al. 1999).

The 1998 outburst. We investigate various models and find that the thermal instability of an accretion disk is the best interpretation of the outburst. This event is of type FRED in accordance with the conditions of the model of King \& Ritter (1998), in which the irradiation by $\mathrm{X}$-rays is strong enough to ionize the entire disk out to its outer edge. The luminosity at the outburst peak is $L_{\text {peak }} \approx 3 \times 10^{38} \mathrm{erg} / \mathrm{s}\left(0.8 L_{\mathrm{Edd}}\right)(d=5 \mathrm{kpc}$ (Robinson et al. 2002)), the disk mass being $M_{\mathrm{h}}(0) \approx 1.5 \times 10^{23} \mathrm{~g}$ and the disk radius being $R_{\mathrm{h}}(0) \approx 2.5 \times 10^{10} \mathrm{~cm}$. These disk parameters are consistent with those of soft X-ray transients (SXTs). More details are given in Šimon et al. (2006). The small $M_{\mathrm{h}}(0)$ and $R_{\mathrm{h}}(0)$ speak in favor of a small, wind-fed disk, in variance with the stream-fed disks in SXTs. A comparison of the absolute magnitude of CI Cam with those of SXTs puts an important constraint on the emission mechanism. The absolute peak magnitude of outburst is $M_{\text {Vout }} \approx-7(d=5 \mathrm{kpc})$. $M_{\text {Vout }}$ of SXTs tends to brighten with the orbital period (Shahbaz \& Kuulkers 1998). A very large disk radius would be needed if it were the site of luminosity in outburst of CI Cam, in contradiction with the small $R_{\mathrm{h}}(0)$. Most luminosity thus comes from a different site, which is supported also by the reddening of the colors in outburst. This can be explained by heating up an extended envelope by a small disk and/or jet formation.

Post-outburst activity of CI Cam. The light curve displays smooth waves on the time scale of hundreds of days, which is in striking variance with the pre-outburst 
fluctuations (Bergner et al. 1995). The color variations are not explicable by the changes of the reddening intrinsic to CI Cam. Significant variations of the continuum must play a role; dominant line(s) changes would lead to rather independent variations of indices. Several superposed spectral components appear to be present. We find the division of the dominant contributions of the spectral components near $\lambda=550 \mathrm{~nm}$ : f-f/f-b emission from the wind and/or envelope (in the red and near-IR passbands; Clark et al. 2000), and another component - (pseudo)photospheric emission - in the blue region. $\mathrm{H} \alpha$ changes are resolved in the colors and suggest that the $\mathrm{H} \alpha$ region evolves on the time scale of hundreds of days. We detect two maxima of a possible 1350 d cycle: (pseudo)photospheric emission, $\mathrm{f}-\mathrm{f} / \mathrm{f}-\mathrm{b}$ emission, and $\mathrm{H} \alpha$ emission are involved in a complicated way in this cycle.

$X$-ray and optical emission in quiescence. We find indication that even if the donor and its wind dominate in the optical in quiescence, they still affect the X-ray flux and Xray absorption $N_{\mathrm{H}}$, measured e.g. by Parmar et al. (2000). Using the Predehl \& Schmitt (1995) relation, the changes of $N_{\mathrm{H}}$ should lead to an increase of $E(B-V)$ by several mag, which is not reflected in the optical colors. Changes of $N_{\mathrm{H}}$ are thus confined to the region hotter than the temperature of the dust condensation and may imply a refilling of the disk embedding the $\mathrm{BH}$. This speaks in favor of the origin of the dominant X-ray emission from the close vicinity of the $\mathrm{BH}$, not from the donor.

Rapid variations in quiescence. A chaotic profile prior to the outburst (Bergner et al. 1995) may suggest under-sampled rapid variations. In any case, the amplitude of the residuals of the night-to-night variations significantly decreased after the outburst. We detect only low-amplitude $(\sim 0.02 \mathrm{mag})$ intra-night variations in four nights; they have a form of waves on the time scale of about an hour. Such rapid changes in B[e] stars may not be related to the presence of the compact object; those in FS CMa were interpreted by de Winter \& van den Ancker (1997) as either accretion events or inhomogeneities in the circumstellar (CM) matter. Since in CI Cam the dust envelope is located too far from the B[e] component (13-52 AU; Robinson et al. 2002), only the inhomogeneities in the CM matter are a viable explanation. As for the non-detection of coherent variations from the compact object, the situation of CI Cam can be similar to the microquasar LS 5039/RX J1826-1450 (Martí et al. 2004). Its absolute mag $M_{\mathrm{V}}=-5.0 \pm 0.3$ (Ribó et al. 2002 ) is close to CI Cam in quiescence. The optical luminosity of the disk embedding the compact object can be out shined by the donor in CI Cam.

This analysis has been supported by the grant 205/05/2167 of the Grant Agency of the Czech Republic.

\section{References}

Belloni, T., Dieters, S., van den Ancker, M. E., Fender, R. P. et al. 1999, ApJ, 527, 345

Bergner, Y. K., Miroshnichenko, A. S., Yudin, R. V., Kuratov, K. S. et al. 1995, A\&AS, 112, 221

Clark, J. S., Miroshnichenko, A. S., Larionov, V. M., Lyuty, V. M. et al. 2000, A\&A, 356, 50

de Winter, D. \& van den Ancker, M. E. 1997, A\&AS, 121, 275

Frontera, F., Orlandini, M., Amati, L., dal Fiume, D. et al. 1998, A\&A, 339, L69

King, A. R. \& Ritter, H. 1998, MNRAS, 293, L42

Martí, J., Luque-Escamilla, P., Garrido, J. L., Paredes, J. M. et al. 2004, A\&A, 418, 271

Parmar, A. N., Belloni, T., Orlandini, M., Dal Fiume, D. et al. 2000, A\&A, 360, L31

Predehl, P. \& Schmitt, J. H. M. M. 1995, A\&A, 293, 889

Ribó, M., Paredes, J. M., Romero, G. E., Benaglia, P. et al. 2002, A\&A, 384, 954

Robinson, E. L., Ivans, I. I. \& Welsh, W. F. 2002, ApJ, 565, 1169

Shahbaz, T. \& Kuulkers, E. 1998, MNRAS, 295, L1

Šimon, V., Bartolini, C., Piccioni, A. \& Guarnieri, A. 2006, MNRAS, 369, 355 